\title{
Research of Products Identity Design of Mechanical Equipment Based on Story Information Element
}

\author{
Huai $\mathrm{Cao}^{1,}$, , Shuting Yang ${ }^{2, b}$ \\ ${ }^{1,2}$ Office No. 415, Dongyi Building, Huazhong University of Science \& Technology, \\ Wuhan, China

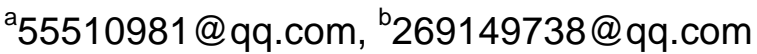

\begin{abstract}
Keywords: mechanical equipment; Product Identity; story; information
\end{abstract}
Abstract. During the product design of a mechanical equipment, story information element could convey Products Identity (PI) more accurate and vivid. After analyzing design requirements, researching and summarizing story information elements, useful design material would be found. The method of expressing story information elements into PI precisely is to construct the story information element mode, summarize systematic and important story information elements, and then building a system of PI. The method is used to instruct the industrial design of the Keso Li-Ion Battery Diaphragm Equipment, which could verify the applicability of story information element to PI design.

\section{Introduction}

PI design is a progress of making enterprise products systematically, which is based on exploring connotation of corporate culture and refining their own characteristics ${ }^{[1]}$. PI design pays attention to its identifiability, unity, and uniqueness, which helps consumers understand product value and connotation of corporate culture. PI design requires multidisciplinary coordination, which should be concerned about both its practical and spiritual value. With the growing of globalization, designers should enhance corporate brand image and competitiveness by digging connotation of corporate culture, refining and using culture symbols synthetically, building cognition to enterprise products.

\section{Function and Influence of Story Information Element}

Human have entered into the information society. Designer could influence people's values and aesthetics ${ }^{[2]}$. Nowadays, people access to information in various way, and information update more rapidly. People become more care about exclusive information, so they become more captious to information selection. Under the premise of meeting the needs, consumers begin to care about the feelings of the product appearance language, interaction between the product and themselves, and also the imagination the product could provide. Product language information can give consumers straightforward materialized image and extend the room for imagination ${ }^{[3]}$.

Story information elements contribute to the transmission of information to help consumers understand the product information, which is the way to connect designers and consumers, so that the function would be understood and used more vividly ${ }^{[4]}$. For PI design, story information element could enhance product affinity, which is not just rational structure, appropriate proportion, and pleasant colors, but also the satisfaction for spirit. Affinity could make consumers have a sense of trust and belonging, which enhance their inner need, such as social statues. Affinity information connect function to appearance organically, reflecting a symbiotic human spirit ${ }^{[5]}$.

Analysis of Story Information Element. The recipient of the information might not know the information element previously ${ }^{[6]}$. So the messages, news, symbols and other forms of information should be conveyed simply and received easily. Story information element is an efficient way to deliver information. People are in uneven distribution on temporal and spatial dimensions when receiving information. This distribution is the existence of matter and energy in conjunction with each other in different regions ${ }^{[7]}$. The amount of story information element can be measured by the amount of 
information. A matter could contain a variety of information elements, through which consumers could perceive the function. Story information element can express either current products existence, or the form of product in the past, and even predict the future trend of development products. Story information element is an effective way to and help communication between consumers and products.

Effect on PI Design of Story Information Element. Story information element is like a bond to PI design ${ }^{[8]}$. Through the transmission of information, consumers could grasp the product features better, understand the product cultural connotation better, and feel the product inner sense better. Story information element has the following effects to PI design:

First, to help consumers understand the product features and styling;

Second, to help consumers perceive the impact of the material of the product;

Third, to help the harmonious relationship between product and environment;

Fourth, to help corporate extract product's overall cultural symbols feature;

Fifth, to help corporate define the symbolic meaning and cultural connotation.

In the progress of PI design, story information embodies the interlinkages and restriction relation among designers, products and consumers: designers giving products information elements, products passing feature information to consumers, and consumers feeding back the information to designers. Dealing with story information elements correctly can guide the PI design effectively. Thus can get a reasonable shape, affinity product features, and sustainable product life cycle. It helps designers and consumers to reach a consensus, to guide the research and design of future products.

Benefit to PI Design of Story Information Element. During PI design, story information elements exist as a valuable substance. People exchange information to interact and communicate, in order to ensure the communication of material and energy in human activities ${ }^{[9]}$. Story information element is a purposeful and regular information exchange process.

PI design makes designers, products, consumers a symbiotic system. Designers seek to elaborate their design concept and functionality of the product through information, which acts as a medium, so that consumers could easily achieve it. Appropriate story information elements make design more mature and easier to accept by consumers. For it serves to both the consumer's satisfaction and the designer's sense of accomplishment. Instead, inappropriate story information would mislead the design intention, or dissatisfy consumer's need of use. Therefore, designers should be innovative and eager to try new products language and method. Besides, designers should identify the universality and particularity of products, focus on product's usage scenario and the product environment-friendly construction, and inject the psychological implications of the information elements into the product. Then, designers could have a comprehensive analysis capability to grasp the most important and characteristic elements among the burdensome types of information. Progress of disposing story information elements is shown in Figure 1.

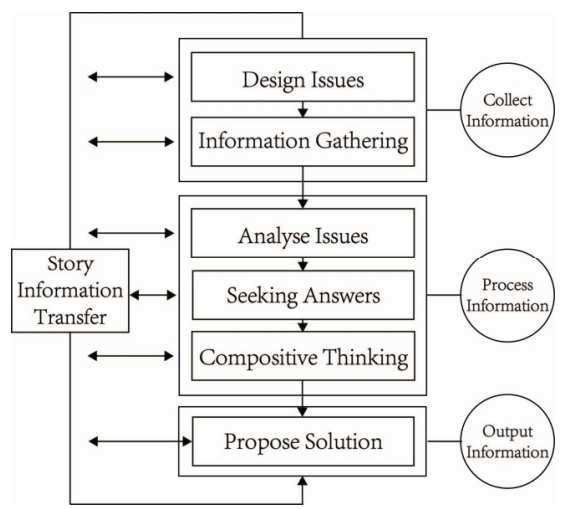

Fig. 1 Process of disposing story information elements 
As shown in Figure 1, story information elements mediate the whole progress of PI design. For designers and the company, it helps find out the design opportunities, define the design objectives, and instruct the development of a complete collection, processing, output, and feedback system. It can also help analyze reasonable design plan in the different stages, exploit the existing market potential and business opportunities. For consumers, it helps comprehend the product functions and spiritual connotation correctly, and have an overall understand of the product's whole life cycle, thus to have a comprehensive evaluation to the product. Then consumers could feedback the usage experience to the designer prosperously. Story information element design is the venation in the product design process, so grasping the story information element means a lot to a reasonable product design approach.

\section{Mode Construction of Story Information Element in PI Design}

Analyze of Basic Elements. Taking PI design of Keso Li-Ion Battery Diaphragm Equipment for example, a story information element model was built from four aspects: shape characteristics specification, color specification system, surface coating specification and interface specification. The framework of the mode could be used in later PI design. A clear product design intent could create a good story information pattern to form a good understanding between the designer and the user, then a clear product positioning could enhance corporate credibility and visibility among consumers to establish a good reputation.

Shape Characteristics Specification. The appearance feature was extracted from the story information elements, and started from "convenient manufacture", "influence of ties" and "intelligent manufacture", which stand for the corporate culture, to refine the constellations, galaxies and star as the story information elements, then comes to the PI design opportunity. For galaxy means extensive and unitive, which is correlated with each other, and full of intelligence and imagination. The message sent by galaxy and constellation agrees with the product spirit. From this, the basic features of modeling were summarized and regulated (as shown in Figure 2).

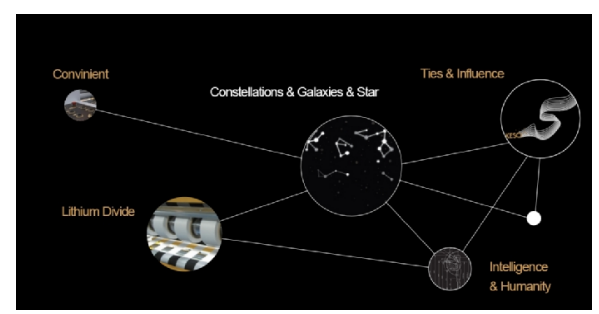

Fig.2 The galaxy story information element of Keso

Constellation flank is an important recognition feature, derived from Keso galaxy. According to different functions of equipment, personal changes of the constellations could be sought in the unified Keso galaxy. The styling specification feature refined constellation flank is shown in Figure 3.

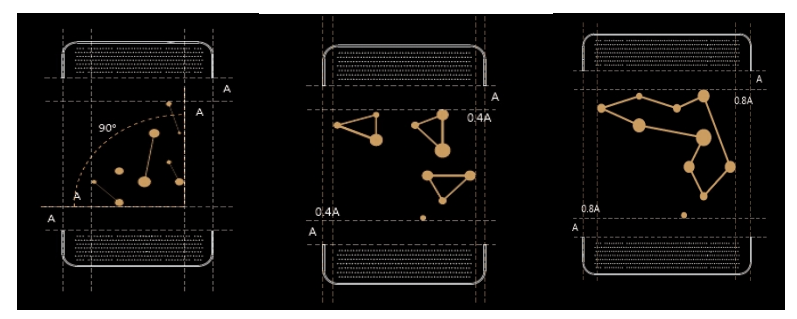

Fig.3 Constellation flank styling specification

Color System Specification. Color system specification includes two parts: standard and secondary color, function and purchased parts reference color. The standard color is refine from the gold color of 
star, and the black color of the night sky. The function color was refined by general standard. Secondary color and reference color should cooperate with the standard color. The specification is shown in Figure 4.

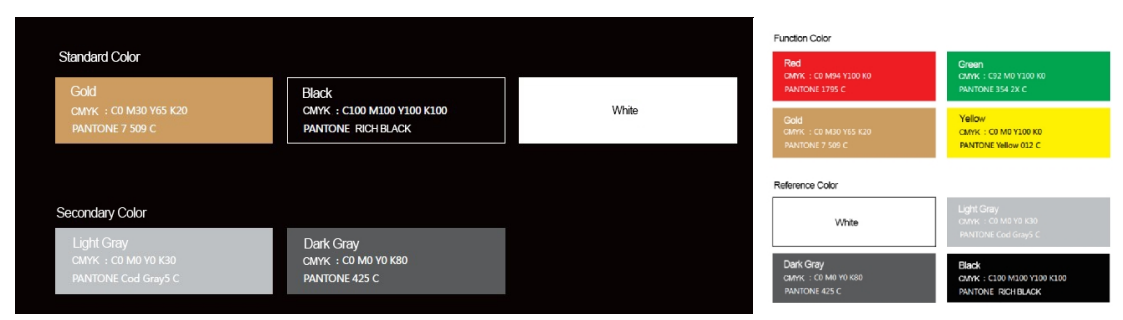

Fig.4 Specification of colors

Surface Coating Specification. Specification of using logo and label model specification can also reflect the story information element. And the safety signs should be stipulated. The specification of surface coating is shown in Figure 5.
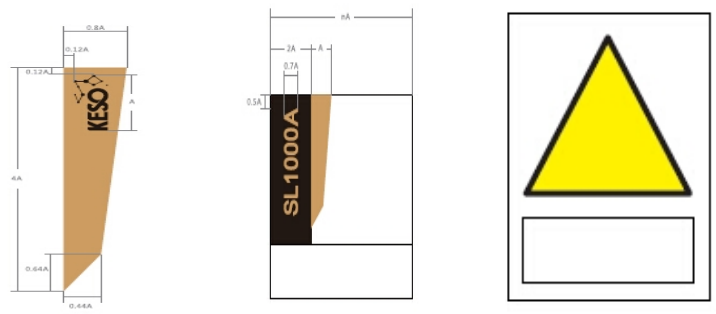

Fig.5 Surface Coating specification

Interface Specification. User interface is an important part of the whole equipment. User interface has the function of human-computer interaction, and people operate the equipment through it, which influence a lot to the user experience. So the user interface is necessary to be easy to use, but also to be unity with the PI design, reflecting its story information elements to some degree. Logo of Keso using specification in the user interface is shown in Figure 6.

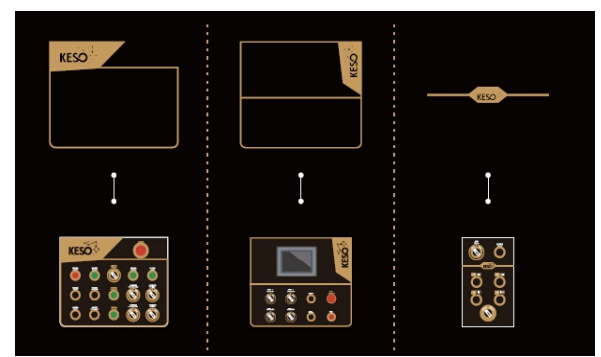

Fig.6 User interface specification

\section{Applications of PI Story Information Element Design Mode}

After analyzing and summarizing story information elements in PI design, and applying the integrated elements into the PI design, a story information element mode is established, which had guided the specific Keso PI design. In accordance with the standards, integrating previous basic elements, and combining with the characteristics of the product itself, the story information elements were extracted to guide the design of splitting machine, hierarchical machine, and compound machine. As shown in Figure 7, the Keso Li-Ion Battery Diaphragm Equipment design have fully embodied the unity and integrity of PI design, as well as its particularity story information elements design, which could be easily to convey the function and corporate inner spirit to the user. 


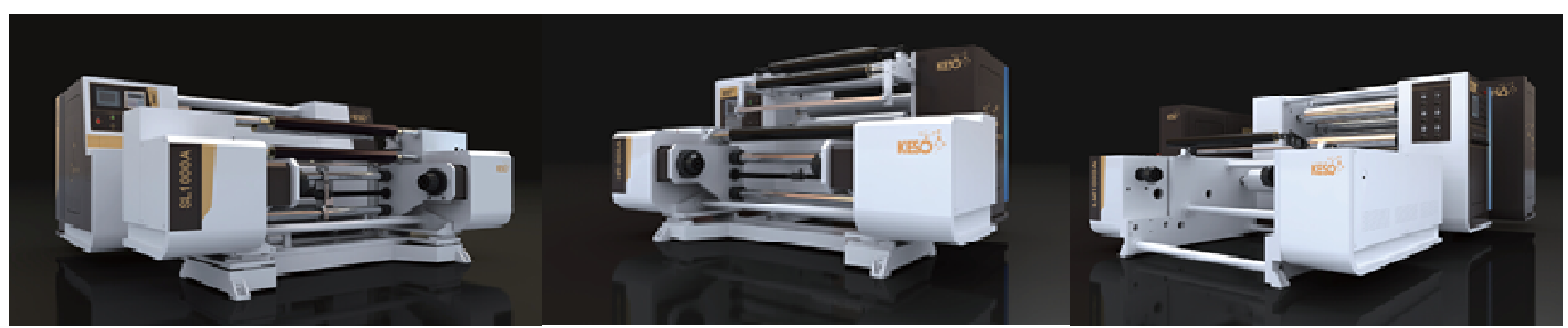

Fig.7 Keso Li-Ion Battery Diaphragm Equipment

\section{Conclusions}

This paper studied on story information element in PI design. Firstly, the impact and role of story information elements and PI design were analyzed. Then, through constructing a mode of story information elements design, the basic elements were proposed to establish a PI system, which is basis for further design. Finally, according to the specification of the story information element design mode, Keso Li-Ion Battery Diaphragm Equipment were designed.

\section{References}

[1] Wei Chen. User Experience Design Elements and Application in Product Design [J]. Packing Engineering, 2011, 32(10):26-29, in Chinese.

[2] Yushan Wang, Shiguo Li: Packing Engineering, 2011, 32(2):56- 59, in Chinese.

[3] Ke Qiu, Minglang Yang, Zhaohong Wan: Packing Engineering, 2012, 33(10):50— 53, in Chinese.

[4] Xianrong Zhang. Design Semiotics. Beijing: Chemical Industry Publishing House , 2004, in Chinese.

[5] Ting Huang: Packing Engineering, 2011, 32(10):18-21, in Chinese.

[6] Junwu Ding, Dongtao Yang, Yadong Cao, et al: Journal of Engineering Design, 2010, 17(1):12 -18 , in Chinese.

[7] Keskin Halit, Byrne John: Product Innovation Management, 2012(3): 31.

[8] Solso Robert L. Cognitive Psychology. Nanjing: Jiangsu Education Publishing House, 2005, in Chinese.

[9] Michalko Michael. How to Diagram and Mind-map Your Thoughts. Creative Thinkering. Novato California: New World Library, 2011. 